\title{
Examination of total cross section resonance structure of niobium and silicon in neutron transmission experiments
}

\author{
Olga Andrianova, Gleb Lomakov ${ }^{\mathrm{a}}$, and Gennady Manturov \\ JSC “SSC RF-IPPE”, Nuclear Reactors and Fuel Cycle Department, 249033 Bondarenko sq. 1, Obninsk, Russia
}

\begin{abstract}
The neutron transmission experiments are one of the main sources of information about the neutron cross section resonance structure and effect in the self-shielding. Such kind of data for niobium and silicon nuclides in energy range $7 \mathrm{keV}$ to $3 \mathrm{MeV}$ can be obtained from low-resolution transmission measurements performed earlier in Russia (with samples of 0.027 to 0.871 atom/barn for niobium and 0.076 to 1.803 atom/barn for silicon). A significant calculation-to-experiment discrepancy in energy range 100 to $600 \mathrm{keV}$ and 300 to $800 \mathrm{keV}$ for niobium and silicon, respectively, obtained using the evaluated nuclear data library ROSFOND, were found. The EVPAR code was used for estimation the average resonance parameters in energy range 7 to $600 \mathrm{keV}$ for niobium. For silicon a stochastic optimization method was used to modify the resolved resonance parameters in energy range 300 to $800 \mathrm{keV}$. The improved ROSFOND evaluated nuclear data files were tested in calculation of ICSBEP integral benchmark experiments.
\end{abstract}

\section{Introduction}

Improvement of Russian national library of evaluated nuclear data files ROSFOND [1] is an actual task for increasing the calculation precision. There are still some incomplete issues in description the resonance structure in niobium and silicon cross-sections in the fast energy region. One of them is a need to validate the niobium unresolved resonance data [2] which are used in deep penetration calculations and in analyses of corresponding experiments. The second task is definition of the $p$-resonance within the energy close to $700 \mathrm{keV}$ viewed in the JEF-2.2 (Fig. 1) but which is not included in new versions of any data libraries.

Testing of the niobium and silicon neutron data in the resonance energy region can be made with help of neutron transmission measurements [3] made through a wide range of thicknesses of samples. The neutron transmission experiments in energy range $1 \mathrm{keV}$ to $3 \mathrm{MeV}$ have been conducted in 1960s for a wide list of reactor materials ( [3]), such as Fe, Ni, Cr, Si, Nb, Be, Cu, Zr, $\mathrm{Mo}, \mathrm{W}, \mathrm{Pb}, \mathrm{Bi}, \mathrm{Al}, \mathrm{Zn}, \mathrm{V}, \mathrm{Ti}, \mathrm{C}, \mathrm{Mg}, \mathrm{P}, \mathrm{S}, \mathrm{Ga}, \mathrm{Sb}$, $\mathrm{Ba}, \mathrm{Ta}$. Transmission experimental data with samples of iron, chromium, and nickel were assessed and included in the ICSBEP Handbook [4] (as FUND-IPPE-VdG-MULTTRANS-001). The integrated within the energy range (see Eq. (1)) measurements were performed by V.Fillipov [5] using the Van de Graff accelerator at the IPPE in Obninsk. The neutron source was generated by a titan-tritium target. Measurements were performed in the energy interval from $1 \mathrm{keV}$ to $3 \mathrm{MeV}$.

\section{Neutron transmission measurements}

The main goal of this work is to test and improve self-shielding effects in the total cross section of these

a e-mail: glomakov@ippe.ru nuclides, because analysis of deep penetration experiments allows finding minima in the $s$-resonance curve which is important.

The measured neutron transmission function in the energy range $\Delta E$ is defined as follows:

$$
T(t)=\int_{\Delta E} R(E) \cdot \exp \left[-\sigma_{t}(E) \cdot t\right] d E,
$$

where $\sigma(E)$ is a value of the total cross section, $t$ is a thickness of a sample and $R(E)$ is an energy spectrum as an experimental resolution function. So-called effective total cross section $\sigma_{\text {eff }}(t)$ for the thickness $t$ in the energy interval $\Delta E$ is defined as follows:

$$
\sigma_{e f f}(t)=\frac{-\ln T(t)}{t} .
$$

The measured neutron transmission functions for each energy interval $\Delta E$, for different thicknesses were converted to the corresponding effective total cross sections and based on these data average total cross sections in different energy intervals were obtained by extrapolation to zero thickness, using a least square procedure, with a good accuracy. Energy intervals were corrected by comparison of experimental and average total cross sections obtained from nuclear data file [6].

\subsection{Niobium experiments}

For niobium two sets of neutron transmission measurement results were obtained for thicknesses of samples of 0.027 to 2.537 atom/barn. The first set of measurements within the energy range 7 to $600 \mathrm{keV}$ with $\Delta E$ intervals $15-40 \mathrm{keV}$; the second one - within the energy range $300 \mathrm{keV}$ to $3 \mathrm{MeV}$ with $\Delta E$ intervals $160-330 \mathrm{keV}$ (the background effect was estimated by measurements with

(c) The Authors, published by EDP Sciences. This is an Open Access article distributed under the terms of the Creative Commons Attribution License 4.0 (http://creativecommons.org/licenses/by/4.0/). 


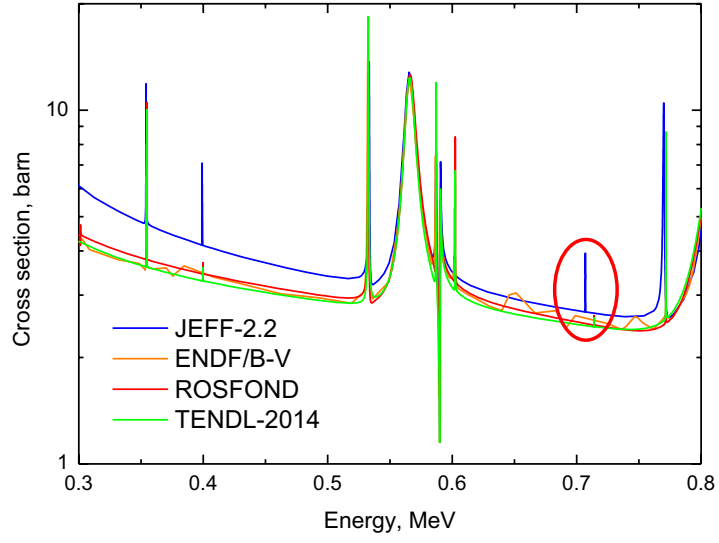

Figure 1. Total cross section of silicon from various libraries in energy range 300 to $800 \mathrm{keV}$.

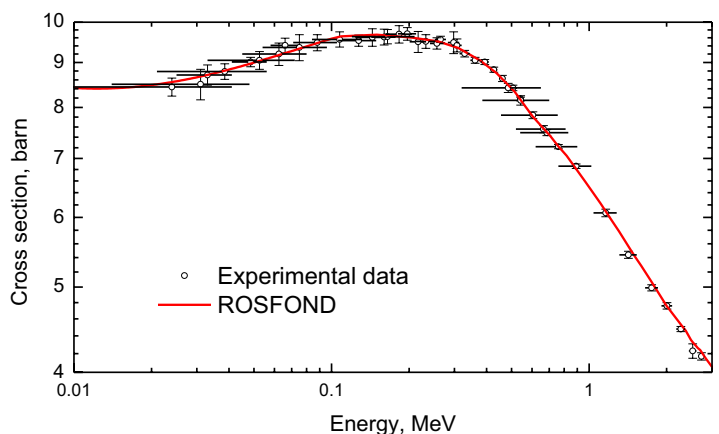

Figure 2. Comparison of the experimental and point-wise (from the ROSFOND) energy averaged total cross sections.

samples of thickness more than 0.9 atom/barn). Evaluation of the transmission functions was obtained for 44 energy intervals. Figure 2 shows a comparison of the estimated total cross sections from experimental energy averaged with origin point-wise total cross sections from the ROSFOND nuclear data library in the energy range from $10 \mathrm{keV}$ to $3 \mathrm{MeV}$.

The values of the total cross section obtained from the measurements of neutron transmission and point-wise ROSFOND nuclear data are well agreed.

Next step was testing of niobium resonance parameters within each energy interval. The interval of energy range 320 to $650 \mathrm{keV}$ (Fig. 3) the most qualitatively shows a necessity to improve parameters of unresolved resonances for niobium. At energies above $600 \mathrm{keV}$ the experimental and evaluated nuclear data for niobium are in a good agreement [7].

\subsection{Silicon experiments}

Neutron transmission measurements for silicon were performed in energy range $300 \mathrm{keV}$ to $3 \mathrm{MeV}$ with interval $160-330 \mathrm{keV}$ for thicknesses of samples of 0.076 to 1.803 atom/barn. Transmission measurements were evaluated in 13 energy intervals [8]. The obtained for silicon effective total cross sections within energy interval from 390 to $700 \mathrm{keV}$ (Fig. 4) in a qualitative manner show a necessity to improve parameters of resolved resonances.

The observed discrepancies show that within the energy range 300 to $800 \mathrm{keV}$ there are inaccuracies in the silicon resonance parameters which included in recent evaluated nuclear data libraries. However, for energies

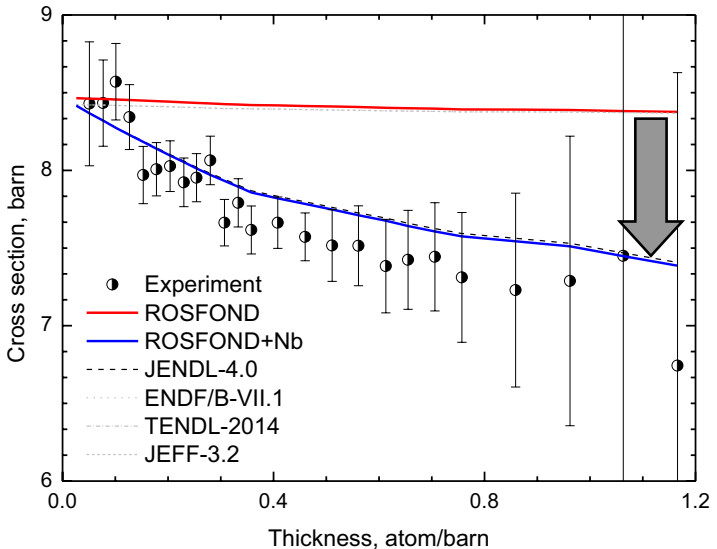

Figure 3. Comparison of niobium effective total cross sections with the data from evaluated nuclear data files in energy range 320 to $650 \mathrm{ke} . \mathrm{V}$

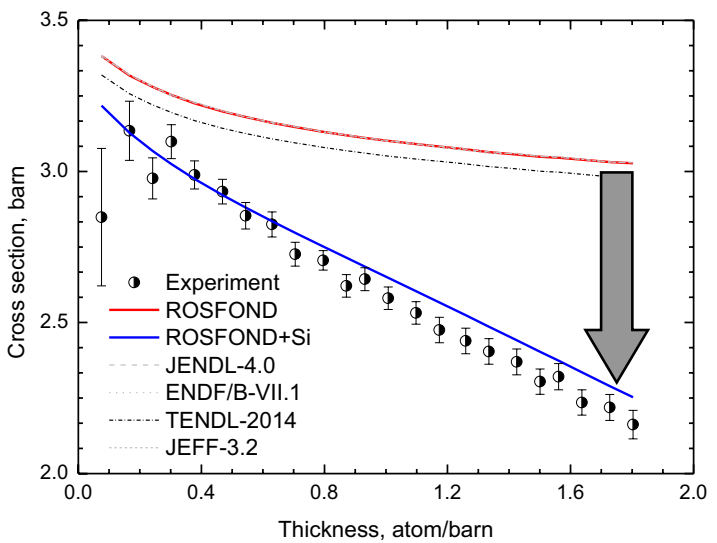

Figure 4. Comparison of silicon effective total cross sections with the data from evaluated nuclear data files in energy range 390 to $700 \mathrm{keV}$.

above $800 \mathrm{keV}$ values of total cross sections obtained from experimental and evaluated nuclear data have well enough agreement [9].

\section{Correction of nuclear data}

The considered transmission measurement results show a necessity for improvement of the niobium and silicon resonance structure parameters, and the ROSFOND nuclear data files (in energy range 100 to $600 \mathrm{keV}$ for niobium and 300 to $800 \mathrm{keV}$ for silicon) were modified based on these measurement results.

\subsection{Niobium data}

The upper energy of resolved resonance parameters ends at about $7 \mathrm{keV}$ in all libraries of evaluated nuclear data. The unresolved resonance region for niobium is absent in ENDF-VII.1 [10] and JEFF-3.2 [11], has upper bounds at $30 \mathrm{keV}$ in TENDL-2014 [12], at $50 \mathrm{keV}$ in BROND3.1 [13], at $100 \mathrm{keV}$ in ROSFOND and at $600 \mathrm{keV}$ in JENDL-4.0 [14]. To improve this situation the EVPAR code [15] was used for estimating for niobium average resonance parameters in energy range 7 to $600 \mathrm{keV}$. New resonance parameters allow minimizing calculated and experimental discrepancies in averaged effective total 
Table 1. Calculation results for HMF-047 benchmark using original and modified niobium ROSFOND data.

\begin{tabular}{cccc}
\hline & Benchmark & ROSFOND & ROSFOND $+\mathrm{Nb}$ \\
\hline $\mathrm{k}_{\text {eff }}$ & $1.0007 \pm 0.0037$ & $1.00497 \pm 0.00025$ & $1.00198 \pm 0.00026$ \\
\hline
\end{tabular}

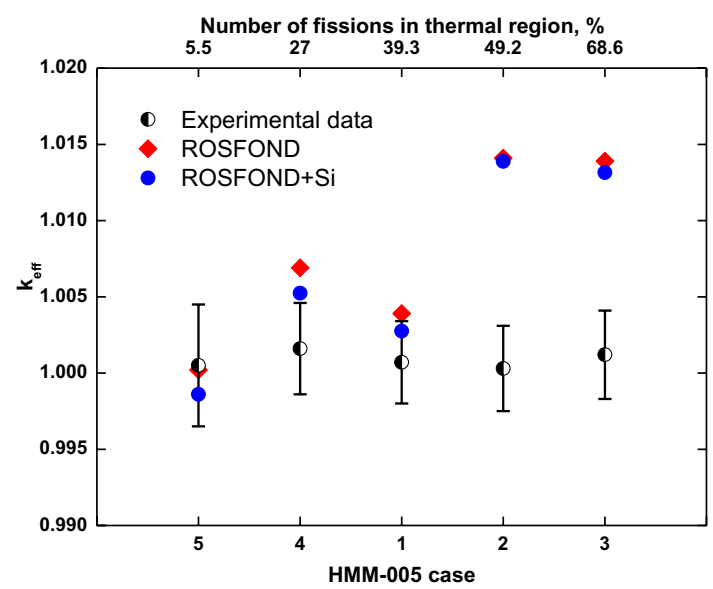

Figure 5. Calculated results of HMM-005 using original and modified silicon ROSFOND data.

cross sections of niobium (see shifting the calculated average cross-section from red to blue curve in Fig. 3).

\subsection{Silicon data}

For silicon all libraries have the same neutron resolved resonance parameters in energy range up to $1.75 \mathrm{MeV}$ [16]. A stochastic optimization method [17] was used for modifying the silicon resolved resonance parameters in energy range 300 to $800 \mathrm{keV}$ which would describe in the best way the experimental curves with regard to minimize discrepancies between the calculated and experimental results (an arrow in Fig. 4 shows the improvement).

The analyzed experimental dependencies of the total transmission measurement functions point to the necessity of revising the adopted estimates of $\mathrm{Si}$ resonance parameters and performing more detailed experiments to investigate neutron cross-section energy dependency in this energy region.

\section{Discussion of results}

The obtained new resonance data for the niobium and silicon were tested through calculations of ICSBEP Handbook integral benchmark experiments: one for niobium (HEU-MET-FAST-047) and two for silicon (HEU-MET-MIXED-005 and PU-MET-MIXED-001).

The HMF-047 experiment was performed and designed to measure the sensitivity of $\mathrm{k}_{\mathrm{eff}}$ to niobium with a softened neutron spectrum such as may occur if a fast-spectrum space reactor core is flooded with water. Calculation results for HMF-047 benchmark using original and modified niobium ROSFOND data are presented in Table 1. They show that the new niobium data improves the $\mathrm{k}_{\mathrm{eff}}$ of HMF-047 benchmark within experimental uncertainty.

The purpose of HMM-005 and PMM-001 experiments was to obtain data that are applicable to a wide range of criticality safety operations involving fissile contaminated

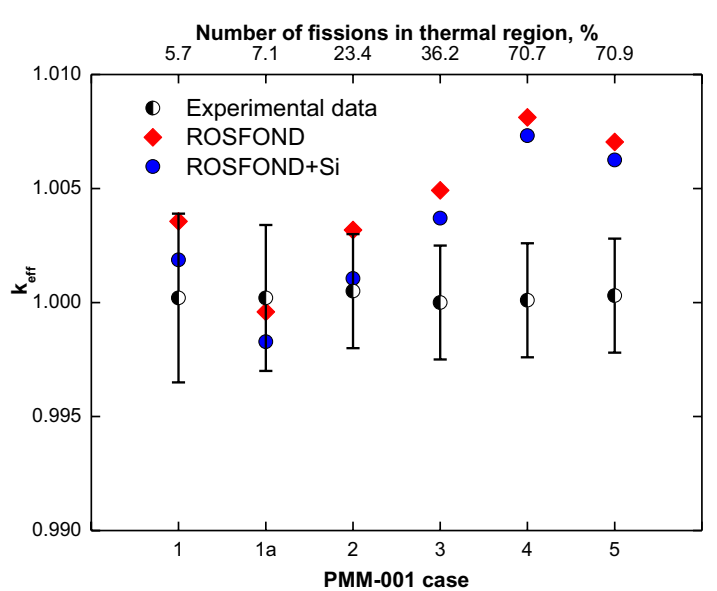

Figure 6. Calculated results of PMM-001 using original and modified silicon ROSFOND data.

wastes. In particular, the data were intended for National Spent Nuclear Fuel Program for validation of criticality safety calculations performed in support of storage of highly enriched spent nuclear fuels in the United States geologic repository at Yucca Mountain (US, Nevada). Results of calculations of uranium (HMM-005) and plutonium (PMM-001) benchmarks using original and modified ROSFOND silicon data are shown in Fig. 5 and Fig. 6 respectively. The new data for the silicon slightly improve the calculation results of the HMM-005 and PMM-001 ICSBEP benchmarks.

\section{Conclusions}

This paper presents results of evaluations previously performed and published data of neutron transmission measurements with niobium and silicon samples of a wide set of thicknesses. The proposed modifications of niobium and silicon resonance parameters for the Russian national library of evaluated nuclear data ROSFOND made it possible to provide a correct agreement between calculation and experimental data, such as the transmission and integral benchmarks.

The objective of the work in this respect was not only to obtain a new evaluation of niobium and silicon resonance parameters but to demonstrate that it is important to develop an understanding of the need for updating of data on the materials under study and to choose neutron cross-sections that adequately describe the resonance selfshielding effects observed in the experiments.

The authors express their gratitude to Dr. M. Nikolaev, Dr. V. Fillipov, Dr. V. Koscheev for fruitful discussions and their kind assistance in carrying out the study.

\section{References}

[1] Zabrodskaya S.V., Ignatyuk A.V., Koshcheev V.N., Manochin V.N., Nikolaev M.N., Pronyaev V.G. PAST. Ser.: NC 1-2, 3 (2007) (available at http://vant .ippe.ru/images/pdf/2007/1.pdf) [in Russian]

[2] Ch. Konno, Yo. Kato, K. Takakura et al., PNST 4, 606 (2014) 
[3] Bondarenko I. I., Nikolaev M.N., Filippov V.V., AE 11, 445 (1961)

[4] International Handbook of Evaluated Criticality Safety Benchmark Experiments, OECD NEA (2011)

[5] Nikolaev M.N., Filippov V.V., Conf: Nuclear Data for Computations (1968)

[6] Lomakov G.B, Nikolaev M.N., Filippov V.V., PAST. Ser.: NRC 1, 148-166 (2016) (available at http: // vant.ippe.ru/images/pdf/2016/1-12.pdf) [in Russian]

[7] Lomakov G.B, Filippov V.V. PAST. Ser.: NRC, 3 (2016, to be published) [in Russian]

[8] Andrianova O., Koscheev V., Lomakov G., Manturov G. PHYSOR 2016, Sun Valley, ID, May 1-5, 2166-2175 (2016)

[9] Lomakov G.B, Filippov V.V. PAST. Ser.: NRC 2, 33-51 (2016) (available at http: //vant .ippe.ru/ images/pdf/2016/2-3.pdf) [in Russian]

[10] Chadwick M.B., Herman M., Oblozinsky P., et al. ND Sheets 112, is. 12, 2887-2996 (2011)
[11] Kopecky J. JEF/DOC-1590

[12] Koning A.J. and Rochman D., ND Sheets 113, 2841 (2012)

[13] Blokhin A.I., Gai E.V., Ignatyuk A.V., Koba I.I., Manokhin V.N., Pronyaev V.G. PAST. Ser.: NRC 2, 62-93 (2016) (available at http://vant .ippe.ru/images/pdf/2016/2-5. pdf) [in Russian]

[14] Shibata K., Iwamoto O., Nakagawa T., Iwamoto N., Ichihara A., Kunieda S., Chiba S., Furutaka K., Otuka N., Ohsawa T., Murata T., Matsunobu H., Zukeran A., Kamada S., Katakura J. NST 48, is. 1, 1-30 (2011)

[15] Manturov G.N., Lunev V.P., Gorbachova L.V., PAST. Ser.: NC 1(50), 50 (1983) [in Russian]

[16] Derrien H., Leal L.C., Larson N.M., Guber K.H., Valentine T.E. and Rauscher T. ORNL/TM-2001/271 (2002)

[17] Andrianova O.N. PhD dissertation (2015) [in Russian] 